AUTHOR'S POST PRINT (Romeo Colour: Green)

Acta Astronautica (IS SN: 0094-5765) Vol. 47, No. 10, pp. 753-761, 2000.

DOI 10.1016/S0094-5765(00)00126-0

Publisher version available at

http://www.sciencedirect.com/science/article/pii/S0094576500001260

\title{
INFLUENCE OF GEOMETRICAL ASPECT RATIO ON THE OSCILLATORY MARANGONI CONVECTION IN LIQUID BRIDGES
}

\author{
R. MONTI, R. SAVINO, M. LAPPA* \\ Università degli Studi di Napoli "Federico II" \\ Dipartimento di Scienza e Ingegneria dello Spazio "Luigi G. Napolitano" \\ P.le V.Tecchio 80, 80125 Napoli (Italy) \\ *current e-mail address: marcello.lappa@strath.ac.uk
}

\section{$\underline{\text { Abstract }}$}

Oscillatory Marangoni convection in silicone oil liquid bridges is investigated by threedimensional, time-dependent numerical solutions of the model equations and by micro-scale experimentation. The field equations are numerically solved with threedimensional control volume methods in a staggered cylindrical non-uniform grid. Two experimental configurations are utilized: 1) the two disks sustaining the bridges are made of copper, their temperatures are controlled with Peltier elements, the flow field in a vertical section is visualized by tracers illuminated by a laser light cut in the meridian plane and four fine temperature sensors are inserted axially from the hot disk into the liquid bridge; 2) the lower disk is made of copper and the upper one is made of transparent glass, heated by an electrical resistance, for visual measurements in a cross section orthogonal to the liquid bridge axis. The surface temperature distribution is measured by an infrared thermocamera. It is shown that the flow field organization, depending on the critical wave number, is related to the geometrical aspect ratio of the liquid bridge and that smaller is the aspect ratio, larger is the critical wave number and more complex the flow-field structure. For each aspect ratio considered, the flow field exhibits a first transition from the axysymmetric steady to a standing wave instability model and then a second transition from the standing wave to the travelling wave regime. The influence of buoyancy effects on the oscillatory Marangoni flow organization is investigated by heating the liquid bridges either from above or from below.

\section{INTRODUCTION}

The stability of Marangoni convection in nonisothermal liquid bridges with quasicylindrical free surfaces has been the object of many researches in the last years. Experiments performed on ground and in microgravity conditions and numerical studies on the subject have been dedicated to the analysis of the transition from the steady axisymmetric toroidal flow to the threedimensional oscillatory flow; this last is established in non isothermal liquid bridges of high Prandtl number liquids when a critical temperature difference across the liquid bridge is exceeded [1-7].

In particular, recent numerical results, substantiated by experiments performed on ground with a micro-scale apparatus [8, 9], have pointed out that, when the temperature difference across the liquid bridge is increased, the flow exhibits two transitions. A first transition occurs from the axi-symmetric steady to a three-dimensional oscillatory state, characterized by a pulsating regime (standing wave model). The second bifurcation is from the pulsating regime to a rotating oscillatory regime (travelling wave model). A flight experiment for a Maxus sounding rocket mission is in preparation and will be performed in fall 1998 for the experimental confirmation of these results, using a silicone oil liquid bridge with length of $2 \mathrm{~cm}$ and an aspect ratio $(\mathrm{A}=\mathrm{L} / \mathrm{D})$ equal to one[10].

In this paper an experimental and numerical analysis is performed to investigate the oscillatory regimes in silicone oil liquid bridges with different aspect ratios under normal gravity. 


\section{EXPERIMENTAL TECHNIQUE}

Liquid bridges of silicone oil with kinematic viscosity $v=2[\mathrm{cs}]$ have been formed and sustained between two cylindrical rods of the same diameter $(\mathrm{D}=4[\mathrm{~mm}])$. The upper disk can be translated along the vertical axis in order to change the geometrical aspect ratio $(A=L / D)$. The translation is obtained using a motorized micrometer system controlled by computer. The volume of the liquid bridge, for all the aspect ratios investigated, is equal to the volume of the cylinder of diameter D and length $\mathrm{L}\left(\pi \mathrm{LD}^{2} / 4\right)$. The temperatures of the bridge supports can be controlled so that an appropriate temperature difference between the upper and lower disk can be imposed and maintained.

The temperatures at four points with the same axial and radial co-ordinate $(\mathrm{x} / \mathrm{L}=0.75$, $\mathrm{r} / \mathrm{R}=0.5$ ) but at different azimuth locations are measured with four thermocouples (with diameter of $0.25[\mathrm{~mm}]$ ) inserted into the microzone through four holes (the thermocouples protrude in the bridge in axial direction).

The liquid motion in the meridian plane is visualized using tracers scattering the light generated by a laser diode (He-Ne with a wavelength of $635 \mathrm{~nm}$ ) forming a light sheet. The laser beam is oriented orthogonal to the main optical path of a CCD camera.

In order to visualize the flow in a section orthogonal to the axis of the bridge a different set up has been utilized with a transparent (glass) upper disk heated by an electrical resistance. The surface temperature distribution is measured by an infrared thermocamera (8-12 $\mu \mathrm{m})$ over an extension of the free surface corresponding to an angular range of about 160 degree.

During an experiment, typically the temperature difference between the end supports is maintained by heating one disk and cooling the other one, with respect to the ambient temperature, with symmetrical temperature ramps. When the Marangoni number is increased a first transition from the axisymmetric to the three-dimensional pulsating regime is observed for $\mathrm{Ma}=\mathrm{Ma}_{\mathrm{c}_{1}}$.
If the Marangoni number is further increased a travelling wave regime is established, characterized by rotating temperature spots along the free surface of the liquid bridge (for $\left.\mathrm{Ma}=\mathrm{Ma}_{\mathrm{c}_{2}}>\mathrm{Ma}_{\mathrm{c}_{1}}\right)$.

The evaluation of the critical Marangoni numbers $\left(\mathrm{Ma}_{\mathrm{c}_{1}}\right.$ and $\left.\mathrm{Ma}_{\mathrm{c}_{2}}\right)$ is made by a rather time consuming procedure, both experimentally and numerically, by identifying a lower and an upper value of Ma at which oscillations occur or do not occur. Reducing the gap between these two values implies long numerical or experimental runs.

The experimental and numerical results described in this paper refer to supercritical flow conditions, i.e. for $\mathrm{Ma}_{\mathrm{c}_{1}}<\mathrm{Ma}<\mathrm{Ma}_{\mathrm{c}_{2}}$ (standing wave) or $\mathrm{Ma}>\mathrm{Ma}_{\mathrm{C}_{2}}$ (travelling wave).

Figs. 1 and 2 show different CCD outputs of a vertical cross-section and a horizontal crosssection in one oscillation period during the pulsating wave regime. Each image corresponds to a CCD frame (the CCD acquisition speed is $25 \mathrm{fps}$ ). The oscillation period $(\tau)$ is of the order of 1 second, the time interval between the different frames is $\tau / 4$. The rotating regime is illustrated in Figs. 3 and 4. The experimental conditions, for this particular case, correspond to an aspect ratio $\mathrm{A}=0.9$, and temperature differences are $20 \mathrm{~K}$, for Figs. 1 and 2, and $40 \mathrm{~K}$, for Figs. 3 and 4. The different behaviour in the pulsating and rotating regimes are more evident in the CCD images, due to the fact that the video acquisition rate is $25 \mathrm{fps}$ (so that there are about 25 frames in each period).

The temperature disturbances are evaluated in a post-analysis phase by subtracting, from the surface temperature distribution, the timeaveraged surface temperature field $T_{0}(z, \varphi)$ obtained integrating the experimentally measured surface temperature distribution over the period $\tau$ of the oscillations. If $\mathrm{N}$ is the number of images taken during the period $\tau$ :

$T_{O}(z, \varphi)=\frac{1}{\tau} \int_{0}^{\tau} T(z, \varphi, t) d t=\frac{1}{N} \sum_{i=1}^{N} T_{i}(z, \varphi)$ 

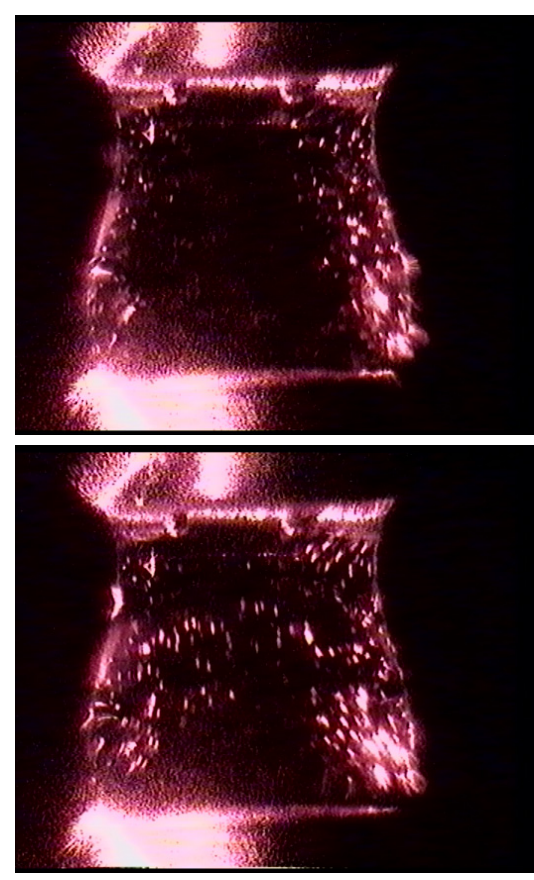

(a) $t=t_{0}$
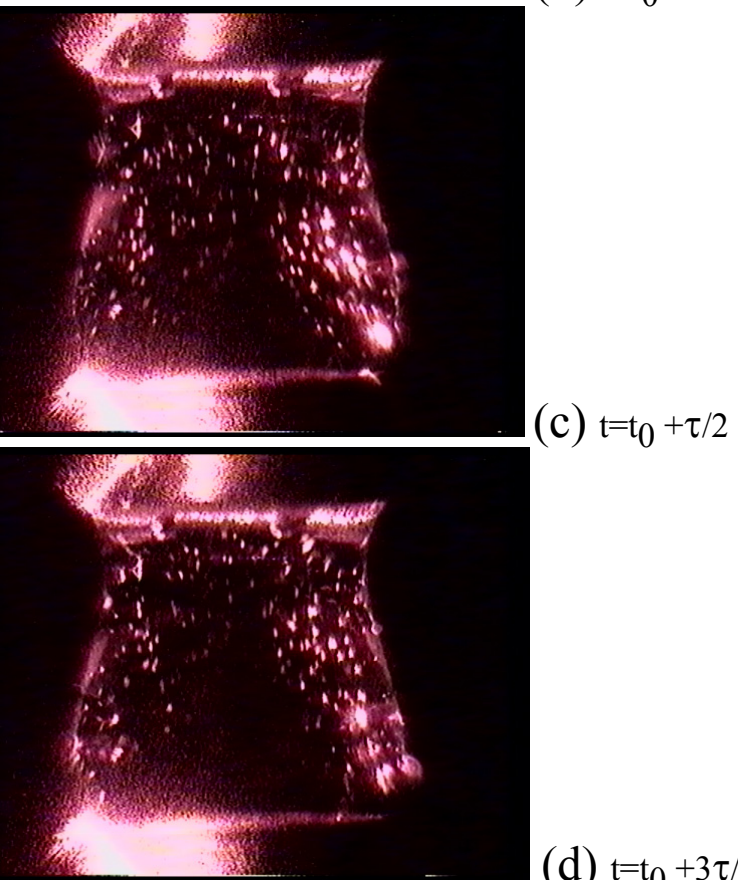

(d) $t=t_{0}+3 \tau / 4$

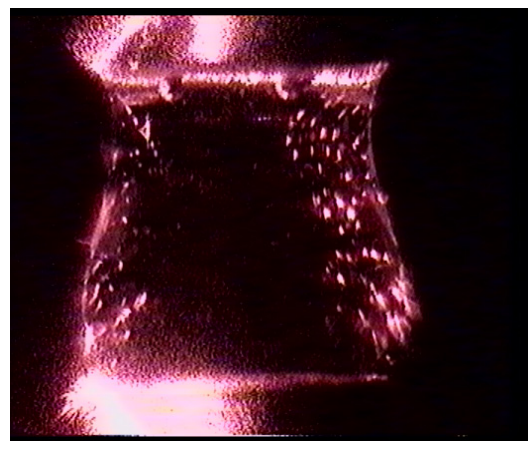

(e) $\mathrm{t}=\mathrm{t}_{0}+\tau$

Fig.1-CCD outputs of the meridian plane in the standing wave regime
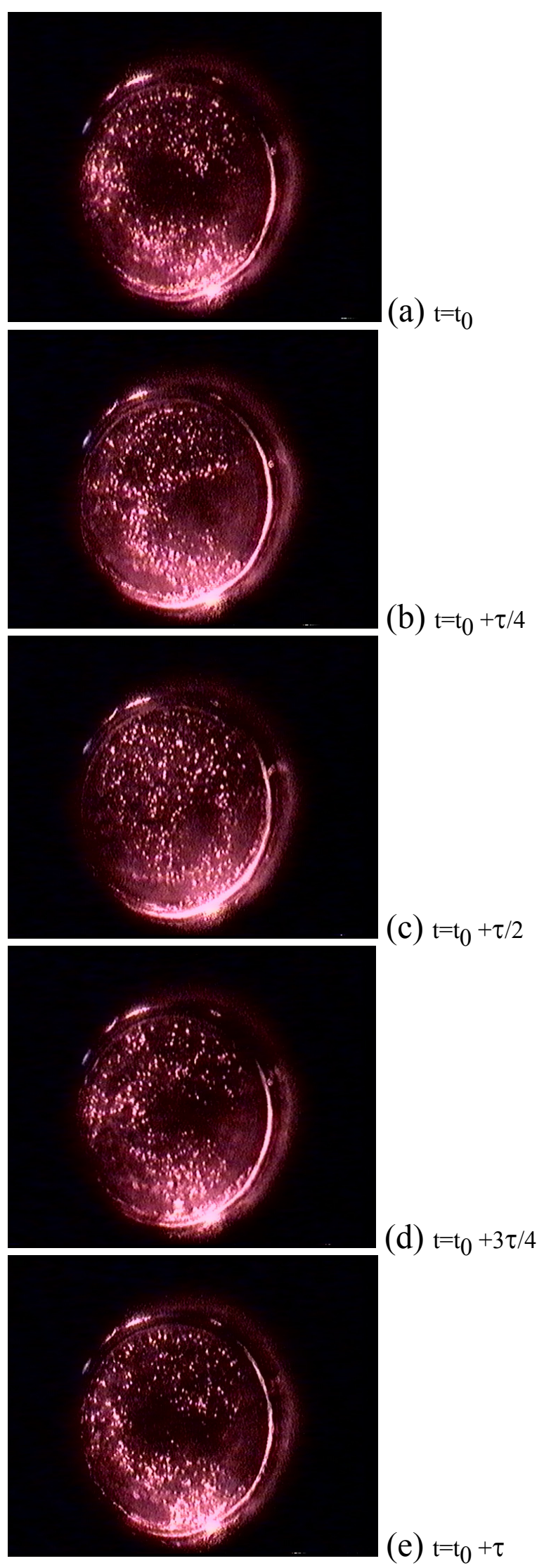

Fig.2 - CCD outputs of the horizontal section in the standing wave regime 


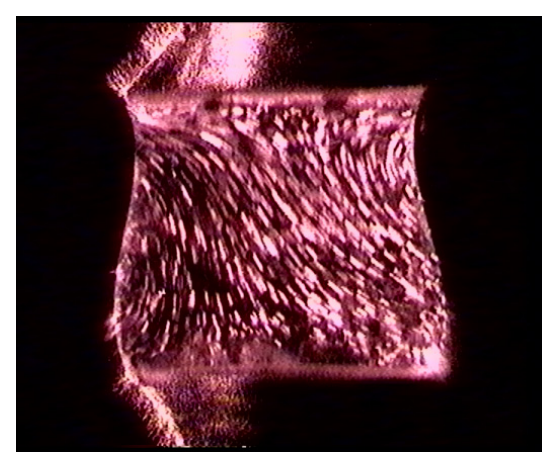

(a) $t=t_{0}$

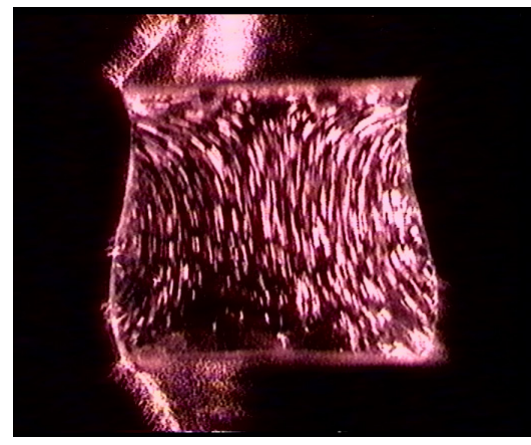

(b) $\mathrm{t}=\mathrm{t}_{0}+\tau / 4$

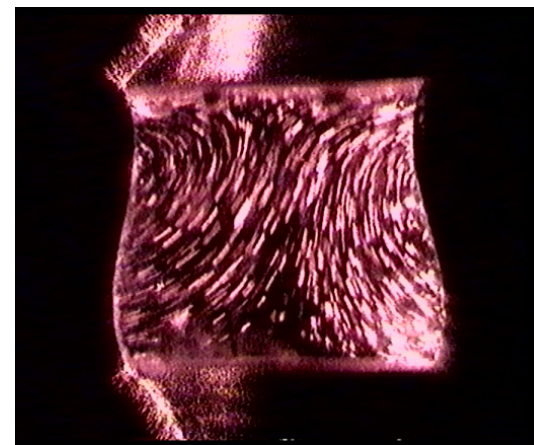

(c) $\mathrm{t}=\mathrm{t}_{0}+\tau / 2$

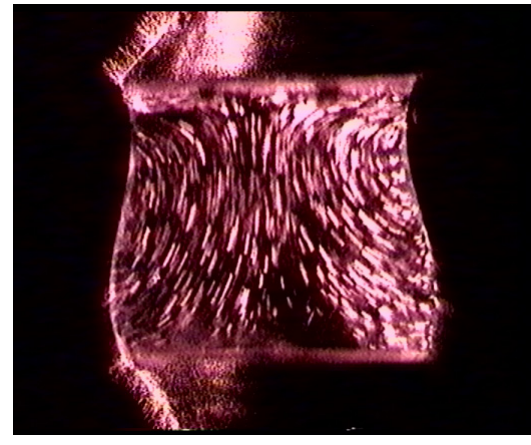

(d) $t=t_{0}+3 \tau / 4$

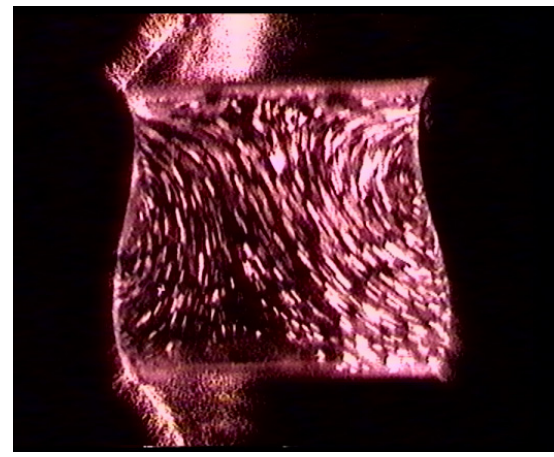

(e) $\mathrm{t}=\mathrm{t}_{0}+\tau$

Fig. 3 - CCD outputs of the meridian plane in the travelling wave regime
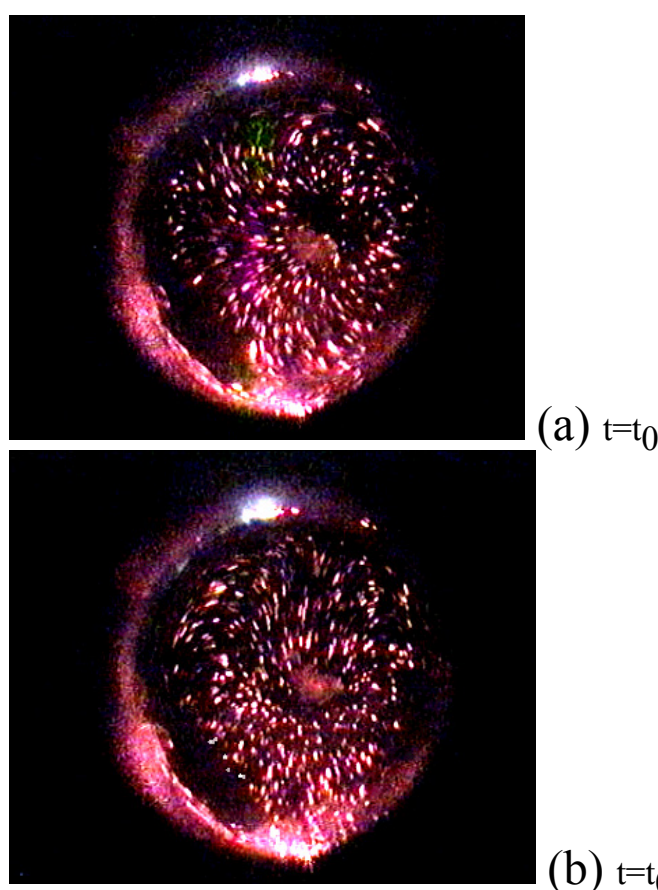

(b) $t=t_{0}+\tau / 4$

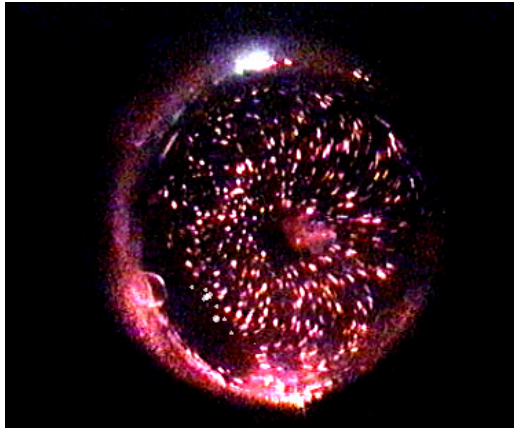

(c) $\mathrm{t}=\mathrm{t}_{0}+\tau / 2$
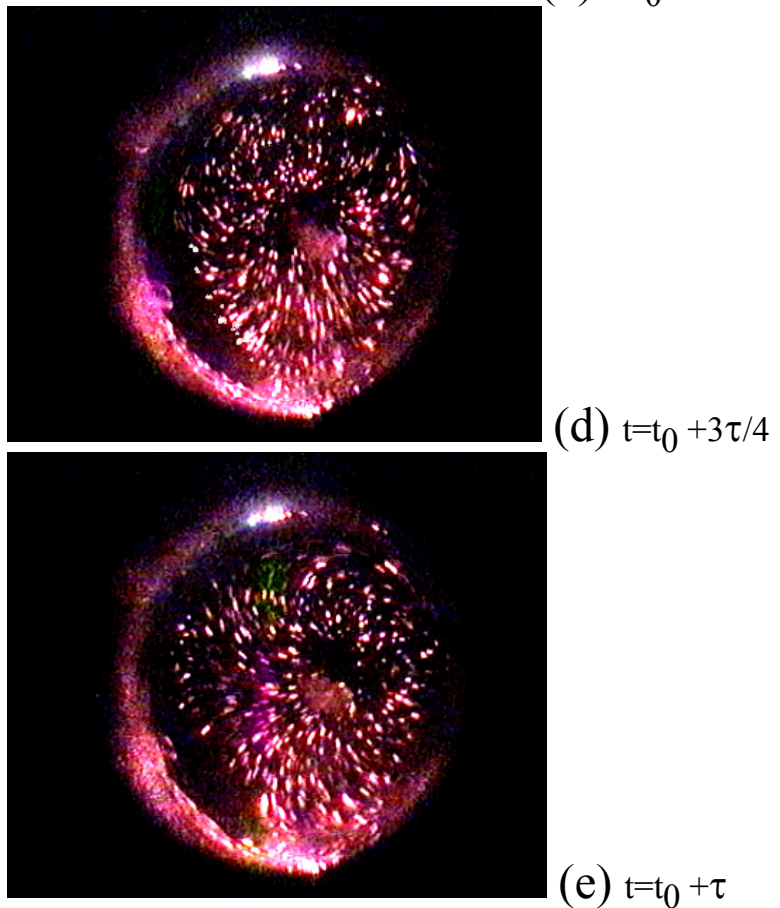

(e) $\mathrm{t}=\mathrm{t}_{0}+\tau$

Fig.4 - CCD outputs of the horizontal section in the travelling wave regime 


\section{NUMERICAL MODEL}

The numerical simulation is performed using a three-dimensional code solving the continuity, Navier-Stokes and energy equations that in non-dimensional conservative form read :

$\underline{\nabla} \cdot \underline{\mathrm{V}}=0$

$\frac{\partial \underline{\mathrm{V}}}{\partial \mathrm{t}}=-\underline{\nabla} \mathrm{p}-\underline{\nabla} \cdot[\underline{\mathrm{V}} \underline{\mathrm{V}}]+\operatorname{Pr} \nabla^{2} \underline{\mathrm{V}}-\operatorname{PrRa} \underline{\mathrm{i}}_{\underline{g}}$

$\frac{\partial \mathrm{T}}{\partial \mathrm{t}}=-\underline{\nabla} \cdot[\underline{\mathrm{VT}}]+\nabla^{2} \mathrm{~T}$

where $\underline{\mathrm{V}}, \mathrm{p}$ and $\mathrm{T}$ are the non-dimensional velocity, pressure and temperature, $\operatorname{Pr}=v / \alpha$ is the Prandtl number, $v$ is the kinematic viscosity and $\alpha$ the thermal diffusivity). The Rayleigh number is defined by $\mathrm{Ra}=\mathrm{g}$ $\beta_{\mathrm{T}} \Delta \mathrm{TL}^{3} / v \alpha$ where $\beta_{\mathrm{T}}$ is the thermal expansion coefficient. The non-dimensional form results from scaling the lengths by the axial distance between the circular disks (L) and the velocity by the energy diffusion velocity $\mathrm{V}_{\alpha}=\alpha / \mathrm{L}$; the scales for time and pressure are, respectively, $\mathrm{L}^{2} / \alpha$ and $\rho \alpha^{2} / \mathrm{L}^{2}$. The temperature is measured with respect to the ambient temperature and scaled by $\Delta \mathrm{T}$. The initial conditions are:

$\mathrm{t}=0: \underline{\mathrm{V}}(\mathrm{z}, \mathrm{r}, \varphi)=\mathrm{T}(\mathrm{z}, \mathrm{r}, \varphi)=0$
For $t>0$, the boundary conditions are the non-slip conditions and the condition of prescribed temperatures on the circular disks, the kinematic condition of stream surface (zero normal velocity), the Marangoni conditions (the reference Marangoni number Ma is defined as $\left.M a=\sigma_{T}(\Delta T) L / \mu \alpha\right)$ and the adiabatic condition on the cylindrical interface. Details on the numerical procedure are given in [8].

\section{RESULTS AND DISCUSSION}

\section{$\underline{4.1 \text { Influence of geometrical aspect ratio }}$}

The experimental and numerical results, in agreement with previous works (see e.g. [3, 4]), pointed out that the temperature and velocity fields, in the oscillatory supercritical state, depend on the geometrical aspect ratio.

Table I summarizes the different experimental configurations, for liquid bridges heated from above. The Marangoni numbers are almost the same for the different aspect ratios considered and correspond to supercritical conditions.

The wave number $\mathrm{m}$, that represents the number of cycles in the azimuth direction [3, $4,8]$ is $\mathrm{m}=1$ for $\mathrm{A}=0.6$, whereas larger values $(m>1)$ have been found for lower aspect ratios. The oscillation frequencies reported in Table I are non dimensional frequencies defined in terms ${ }_{3)}$ of the characteristic frequency $\mathrm{f}_{\mathrm{c}}=\alpha / \mathrm{D}^{2}$.

\begin{tabular}{|c|c|c|c|c|c|c|}
\hline $\mathrm{A}$ & $\mathrm{Ra}$ & $\mathrm{Ma}$ & $\mathrm{m}$ (exp.) & $\mathrm{m}$ (num) & $\begin{array}{c}\mathrm{f} \times 10^{-2} \\
\text { (exp.) }\end{array}$ & $\begin{array}{c}\mathrm{f} \times 10^{-2} \\
\text { (num.) }\end{array}$ \\
\hline 0.25 & $7.210^{3}$ & $3.810^{4}$ & 3 & 3 & 8.9 & 9.4 \\
\hline 0.4 & $1.6710^{4}$ & $3.610^{4}$ & 2 & 2 & 5.4 & 3.6 \\
\hline 0.5 & $2.5210^{4}$ & $3.510^{4}$ & 2 & 2 & 4.1 & 3.2 \\
\hline 0.7 & $4.910^{4}$ & $3.210^{4}$ & 1 & 1 & 3.3 & 2.5 \\
\hline 0.9 & $8.110^{4}$ & $3.510^{4}$ & 1 & 1 & 2.2 & 2.0 \\
\hline
\end{tabular}

Table I: Experimental and numerical results (bridge heated from above)

The flow structure of the supercritical state is related to the value of $\mathrm{m}$ and hence depends on the value of the aspect ratio. Higher is $m$, more complex is the flow organization. Figs 5,6,7 show for each aspect ratio the temperature disturbances on the liquid bridge surface (Figs. 5a, 6a and 7a refer to the experimental results, Figs. $5 \mathrm{~b}, 6 \mathrm{~b}$ and $7 \mathrm{~b}$ to the numerical results).

For the case shown in Figs. 5 (corresponding to $\mathrm{A}=0.7$ and $\mathrm{Ma}=3.2 \times 10^{4}$ ) there are two temperature spots (one hot and the other cold) along the entire free surface of the liquid bridge and hence $\mathrm{m}=1$. The experimental 
temperature distribution over a surface portion of about 160 degrees shows in fact only the hot spot.

The case $\mathrm{m}=2$ (corresponding to $\mathrm{A}=0.4$ and $\mathrm{Ma}=3.610^{4}$ ) is illustrated in Figs.6.

These numerical results show the presence of four temperature spots (two cold and two hot) along the entire surface, whereas two thermal spots (one hot and the other cold) appear in the thermocamera image. The case $\mathrm{m}=3$ is shown in Figs. $7(\mathrm{~A}=0.25$ and $\mathrm{Ma}=3.8$ $\left.10^{4}\right)$.

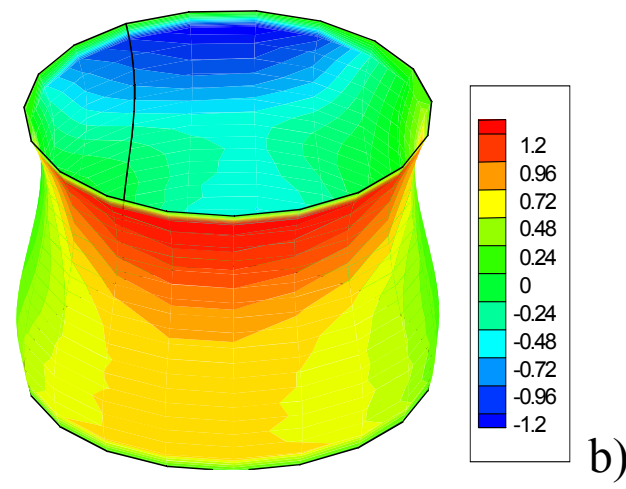

Fig. 5 - Surface temperature disturbances for $A=0.7, M a=3.210^{4}$. (a): experimental; (b): numerical
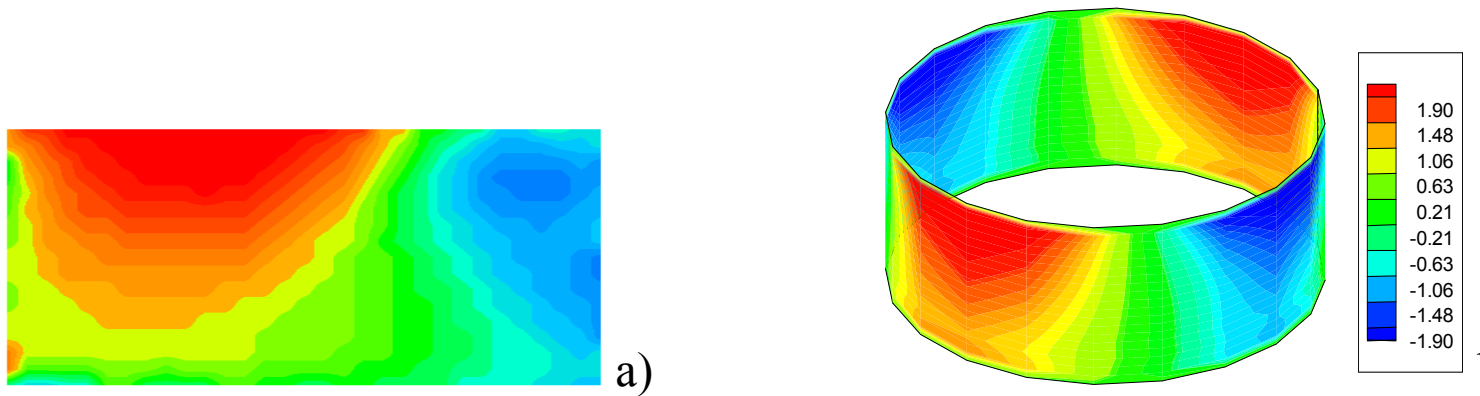

b)

Fig. 6 - Surface temperature disturbances for $A=0.5, M a=3.610^{4}$. (a): experimental; (b): numerical
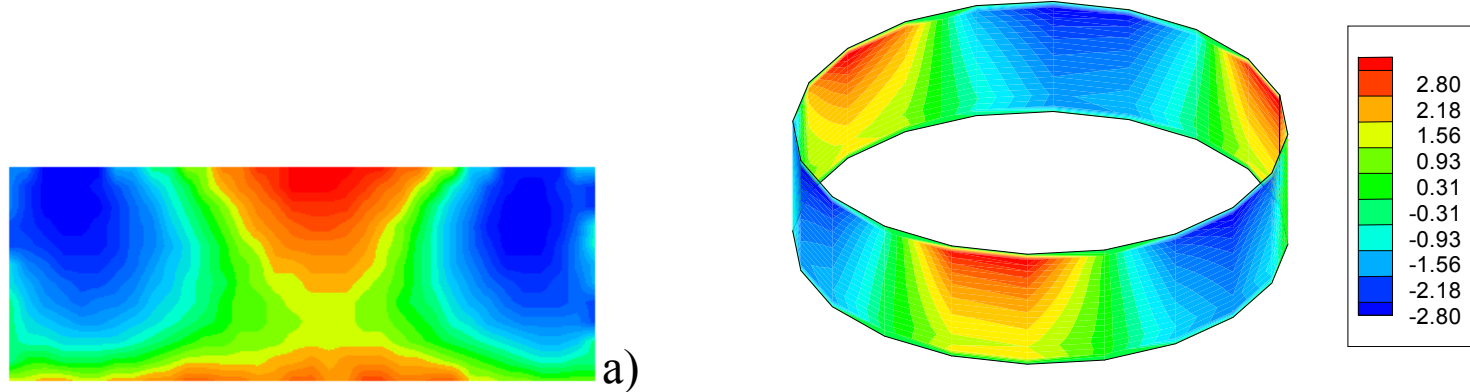

b)

Fig. 7 - Surface temperature disturbances for $A=0.25,3.810^{4}$. (a): experimental; (b): numerical

Odd wave numbers (e.g. $\mathrm{m}=1$ and $\mathrm{m}=3$ ) correspond to "asymmetrical" modes; even values of $m($ e.g. $m=2)$ correspond instead to "symmetric" modes. More generally, when the critical disturbance number (m) is odd, there are two asymmetrical vortex cells in each meridian plane of the liquid bridge.

In particular for $m=1$ the supercritical oscillatory flow appears as an inclined toroidal vortex, as shown by Figs. 8 and 9, that show the experimental and numerical vector plots in the vertical and horizontal cross sections, for the case $\mathrm{A}=0.9, \mathrm{Ma}=3.5$ $10^{4}$. The vortex in one half of the zone section appears smaller than the opposite vortex. The time-dependence is observed as periodical interchange of the shape of the vortices in the left and right parts of the zone. After one half of the oscillation period the small vortex and the large vortex change 
position. The branching streamline of the opposite vortices changes its inclination continuously in an oscillation period (Fig. 8).

For even critical wave numbers, the flow field structure is on the whole three-dimensional and depends on the azimuth co-ordinate, but in each axial plane the velocity and the temperature fields are symmetric and the time-dependence is observed as a synchronous pulsation of the two symmetrical vortices.

This behaviour is illustrated in Figs. $10(\mathrm{~A}=$ $0.5, \mathrm{~m}=2)$. The velocity field in this case is symmetric and the convective cells travel axially up and down.

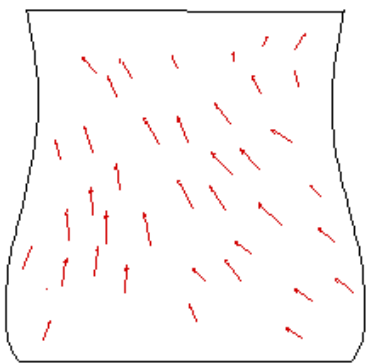

(a): experimental

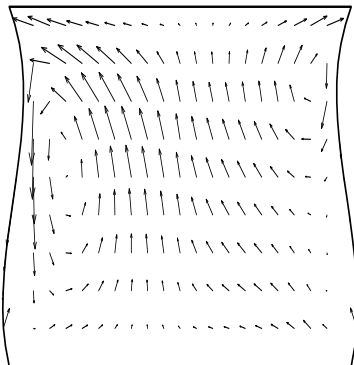

(b): numerical

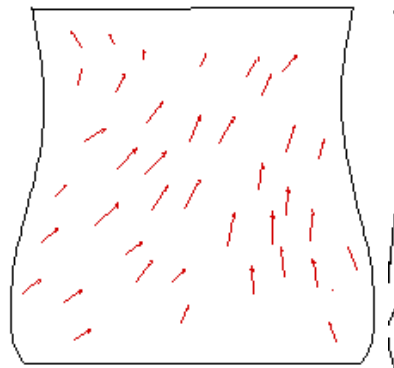

(c): experimental

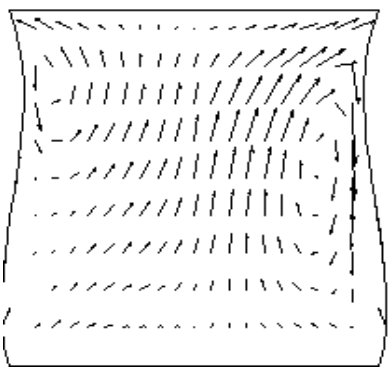

(d): numerical
$0.6 \mathrm{~cm} / \mathrm{s}$

Fig. 8- Vector plots in the meridian plane at two times (half period apart) in the oscillation period $\left(\mathrm{A}=0.9, \mathrm{Ma}=3.210^{4}\right)$

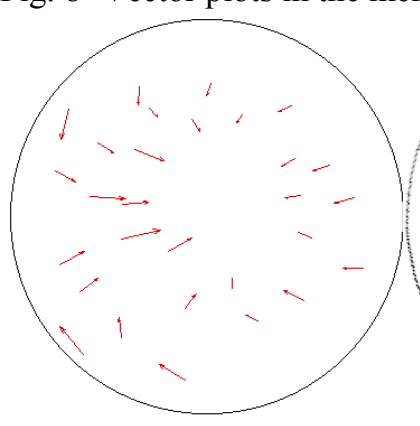

(a): experimental

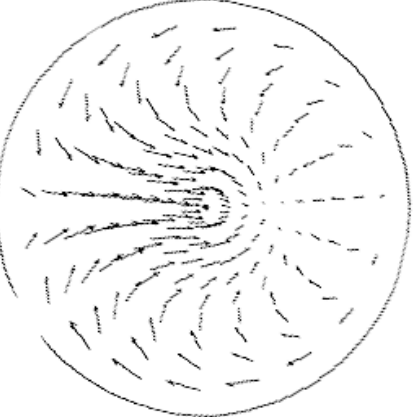

(b): numerical

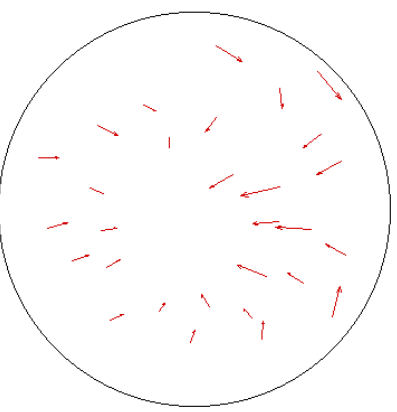

(c): experimental

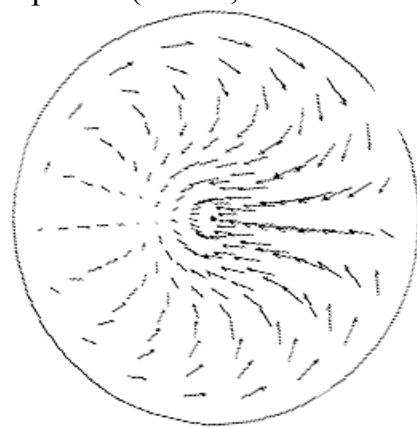

(d): numerical

Fig. 9- Vector plots in the cross section at two times (half period apart) in the oscillation period $\left(\mathrm{A}=0.9, \mathrm{Ma}=3.210^{4}\right)$

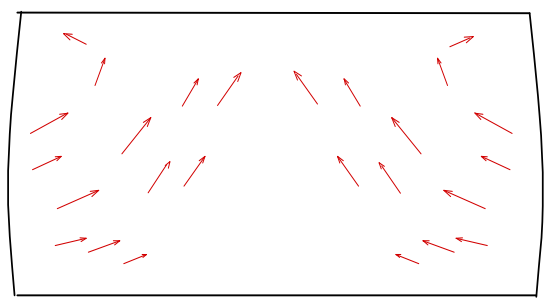

(a): experimental

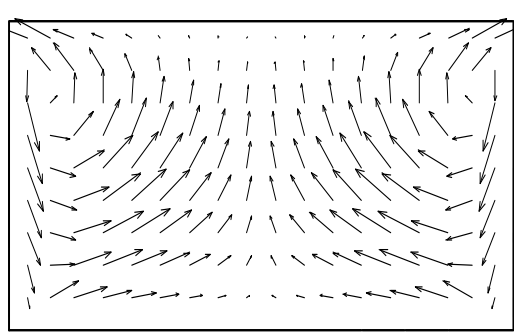

(d): numerical

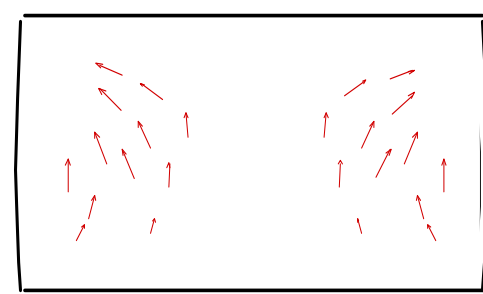

(b): experimental

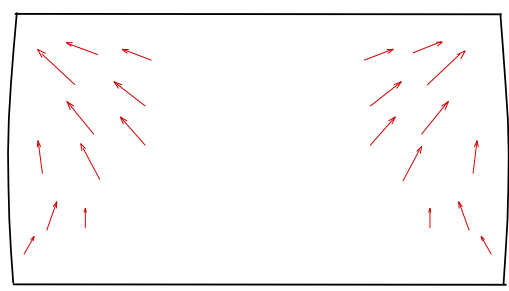

(c): experimental

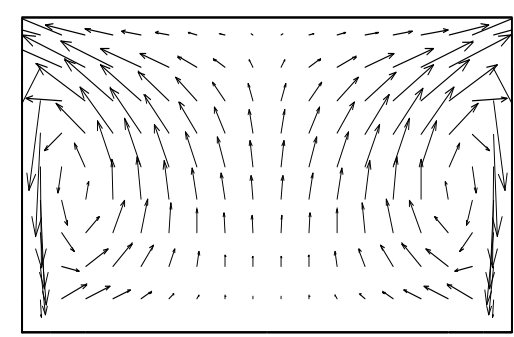

(f): numerical

$0.3 \mathrm{~cm} / \mathrm{s}$

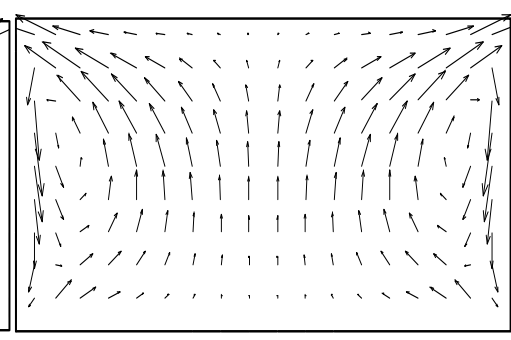

(e): numerical
$0.7 \mathrm{~cm} / \mathrm{s}$

Fig. 10- Vector plots in the meridian plane at two times (one fourth period apart) in the oscillation period $\left(\mathrm{A}=0.5, \mathrm{Ma}=3.510^{4}\right)$ 


\section{$\underline{4.2 \text { Pulsating and rotating regimes }}$}

In agreement with previous works [8-10], the experimental and numerical results confirmed that, for all the aspect ratios considered, immediately after the onset (for $\mathrm{Ma}>\mathrm{Ma}_{\mathrm{c}}$ ), an "azimuthally standing wave" is formed. The three dimensional temperature disturbance consists of a number $m$ of couple of spots (hot and cold) "pulsating" at the same azimuth positions along the interface (minimum and maximum disturbances at fixed azimuth positions). If the Marangoni number is further increased, a travelling wave appears, characterized by rotating temperature spots along the free surface of the liquid bridge (for $\mathrm{Ma}=\mathrm{Ma}_{\mathrm{c}_{2}}>\mathrm{Ma}_{\mathrm{c}_{1}}$ ).

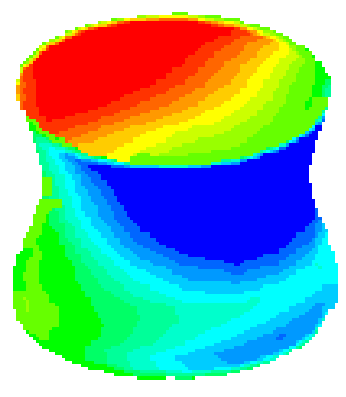

(a): $\mathrm{t}=$ to

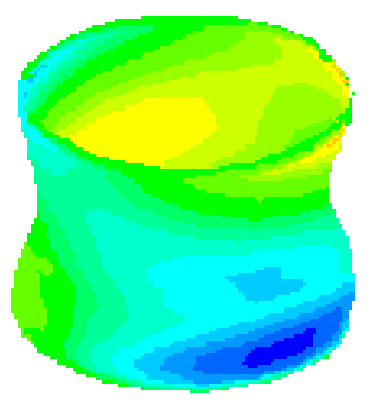

(b): $\mathrm{t}=\mathrm{to}+\tau / 6$

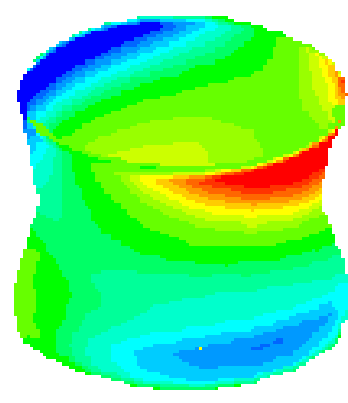

(c): $\mathrm{t}=\mathrm{to}+\tau / 3$
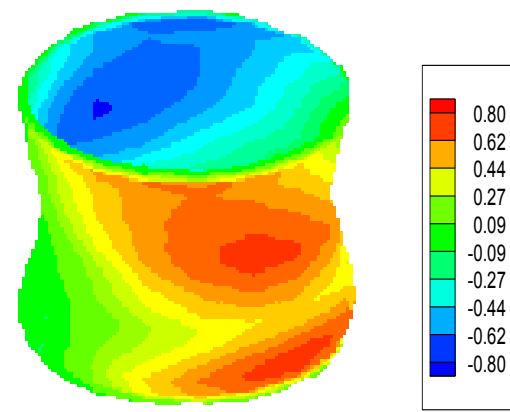

(d): $\mathrm{t}=\mathrm{to}+\tau / 2$

Fig. 11- Numerical surface temperature disturbances in half oscillation period $\left(\mathrm{A}=0.9, \mathrm{Ma}=3 \times 10^{4}\right.$, pulsating)

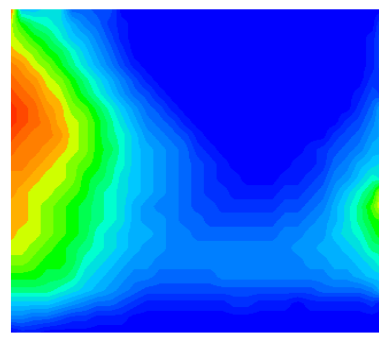

(a): $\mathrm{t}=$ to

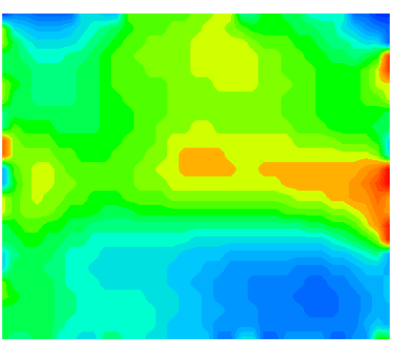

(b): $\mathrm{t}=\mathrm{to}+\tau / 6$

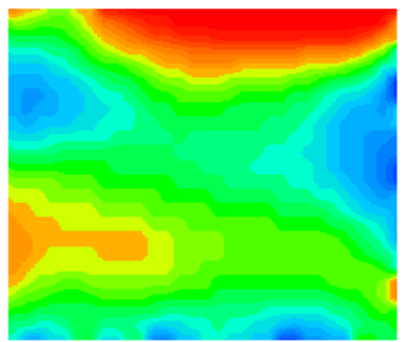

(c): $\mathrm{t}=\mathrm{to}+\tau / 3$

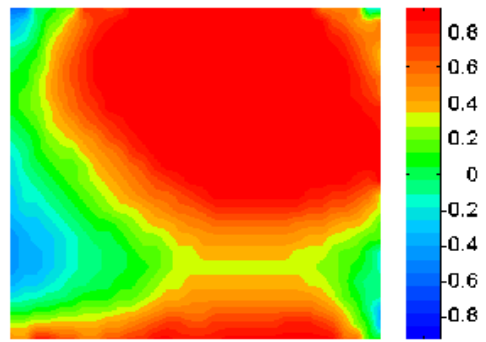

(d): $\mathrm{t}=\mathrm{to}+\tau / 2$

Fig. 12-Experimental surface temperature disturbances in half oscillation period $\left(A=0.9, \mathrm{Ma}=3 \times 10^{4}\right.$, pulsating $)$

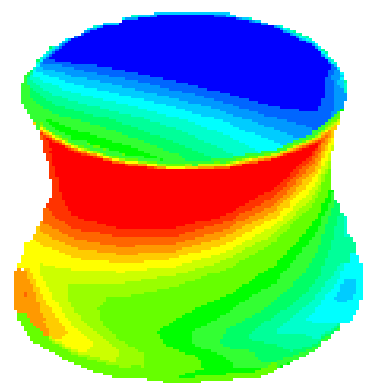

(a): $\mathrm{t}=$ to

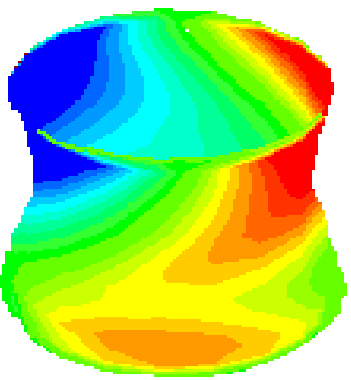

(c): $\mathrm{t}=\mathrm{to}+\tau / 2$

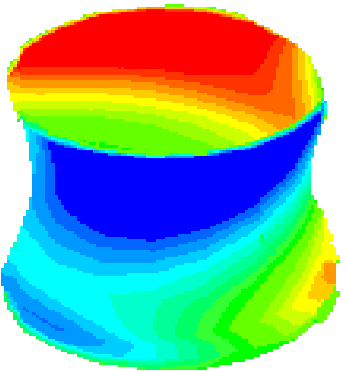

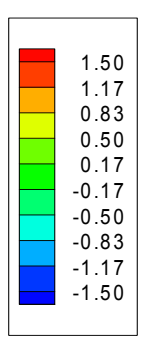

(d): $\mathrm{t}=\mathrm{to}+(3 / 4) \tau$

Fig. 13-Numerical surface temperature disturbances in the oscillation period $\left(\mathrm{A}=0.9, \mathrm{Ma}=3.5 \times 10^{4}\right.$, rotating) 


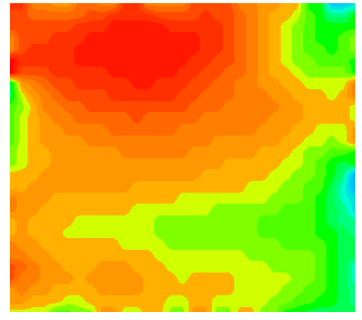

(a): $\mathrm{t}=$ to

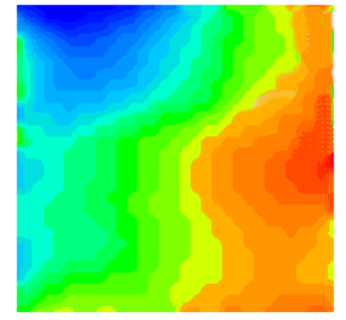

(b): $\mathrm{t}=\mathrm{to}+\tau / 4$

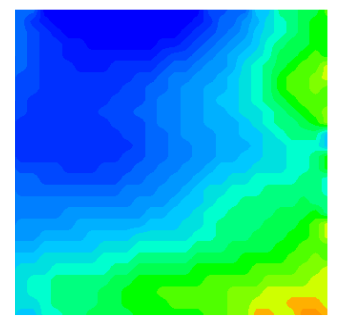

(c): $\mathrm{t}=\mathrm{to}+\tau / 2$

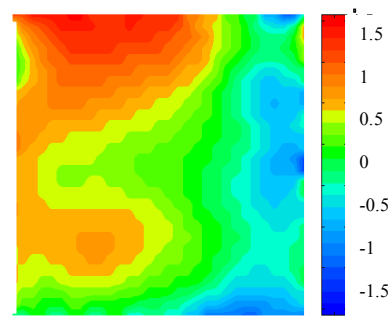

(d): $\mathrm{t}=\mathrm{to}+(3 / 4) \tau$

Fig. 14-Experimental surface temperature disturbances in the oscillation period $\left(\mathrm{A}=0.9, \mathrm{Ma}=3.5 \times 10^{4}\right.$, rotating $)$

Figs. 11 and 12 show, respectively, the computed and measured surface temperature distributions with pulsating temperature spots during the regime of standing wave for $\mathrm{A}=0.9$ $\left(\mathrm{Ma}_{\mathrm{c}_{1}}<\mathrm{Ma}<\mathrm{Ma}_{\mathrm{c}_{2}}\right)$. The rotating behaviour during the travelling wave regime is illustrated in Figs. 13 and $14\left(\mathrm{Ma}>\mathrm{Ma}_{\mathrm{c}_{2}}\right)$.

The appearance of an azimuthally standing wave that bifurcates in an azimuthally travelling wave has been observed for all the aspect ratios considered, but the standing wave model is more stable for small aspect ratios. For $m=1$ the transition from the standing wave to the travelling wave regime has been also observed by the behaviour of the temperature time profiles detected by the four thermocouples located at different azimuth positions in the same horizontal cross section (see Figs. 15). As discussed in [10], in the standing wave regime there is no phase shift between the signals detected by two thermocouples with an azimuth shift of $90^{\circ}$ and the phase shift between two thermocouples at $180^{\circ}$ is $\pi$; in the travelling wave regime the phase shift between two consecutive thermocouples at $90^{\circ}$ is $\pi / 2$.

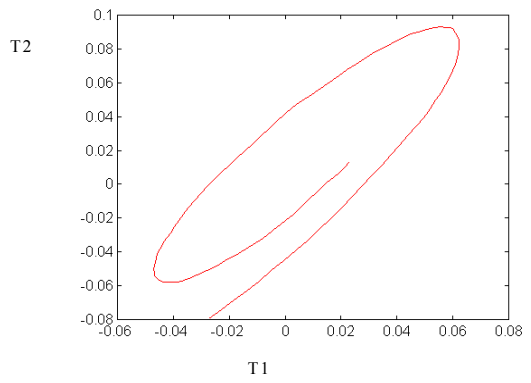

(a): numerical, standing wave

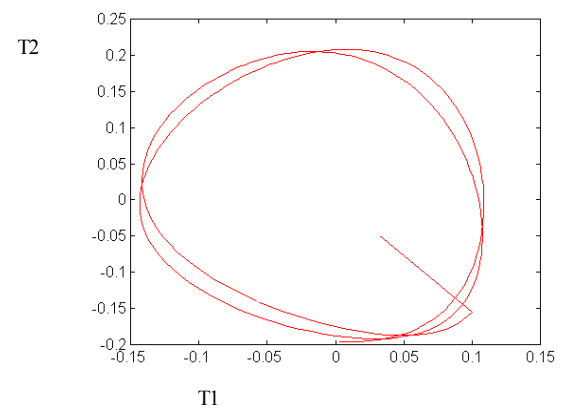

(c): numerical, travelling wave
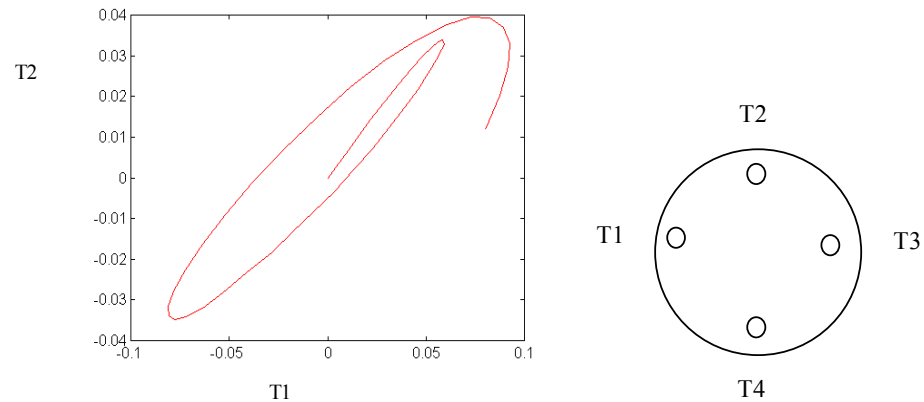

(b): experimental, standing wave
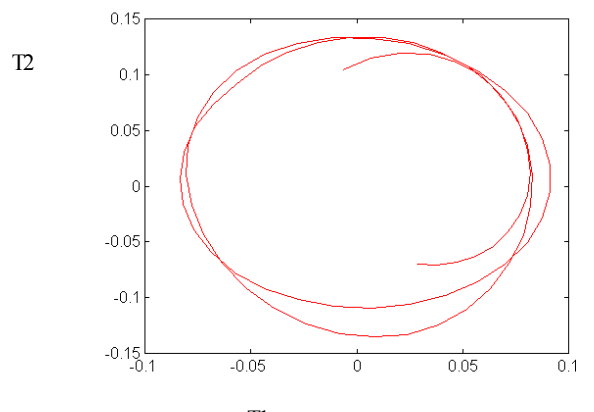

$\mathrm{T} 1$

Fig. 15- Temperatures detected by thermocouples in a cross section (conditions of Figs. 11-14) 


\subsection{Influence of buoyancy effects}

The influence of buoyancy effects on the onset of Marangoni instability and on the flow organization has been investigated at different aspect ratios, by heating the bridge from below or from above. The experimental conditions investigated, for liquid bridges heated from below, are summarized in Table II.

Comparison with Table I shows that the Marangoni numbers, corresponding to oscillatory supercritical conditions, are slightly increased (compared to the case of bridges heated from above). This increase is more evident for high aspect ratios since the Rayleigh number (which measures the intensity of the buoyancy forces) increases proportionally to $\mathrm{L}^{3}$.

The azimuth flow organization, related to the critical wave number $\mathrm{m}$, may be different according to whether the bridge is heated from above or from below.

\begin{tabular}{|c|c|c|c|c|c|c|}
\hline $\mathrm{A}$ & $\mathrm{Ra}$ & $\mathrm{Ma}$ & $\mathrm{m}$ (exp.) & $\mathrm{m}$ (num) & $\begin{array}{c}\mathrm{f} \times 10^{-2} \\
(\mathrm{exp} .)\end{array}$ & $\begin{array}{c}\mathrm{f} \times 10^{-2} \\
(\text { num. })\end{array}$ \\
\hline 0.25 & $7.6510^{3}$ & $4.210^{4}$ & 2 & 2 & 8.6 & 7.8 \\
\hline 0.4 & $2.0310^{4}$ & $4.410^{4}$ & 1 & 1 & 4.9 & 3.4 \\
\hline 0.5 & $3.110^{4}$ & $4.310^{4}$ & 1 & 1 & 3.4 & 2.9 \\
\hline
\end{tabular}

Table II: Experimental and numerical results (bridge heated from below)

The numerical and experimental surface temperature distributions obtained in the case $\mathrm{A}=0.4$, are illustrated in Figs. 16. The number of the temperature spots on the liquid bridge surface, provided by the thermocamera and the corresponding numerical results confirm that at the critical conditions the wave number

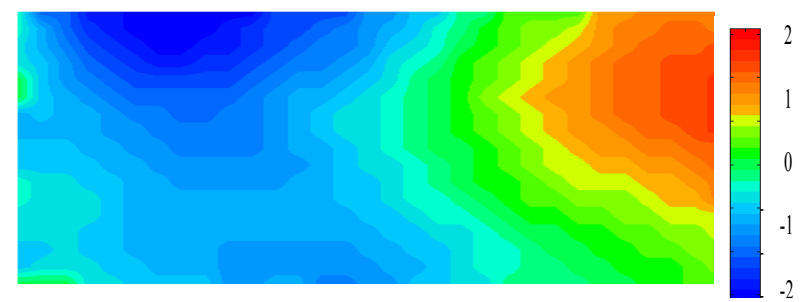

(a): experimental, heating from above

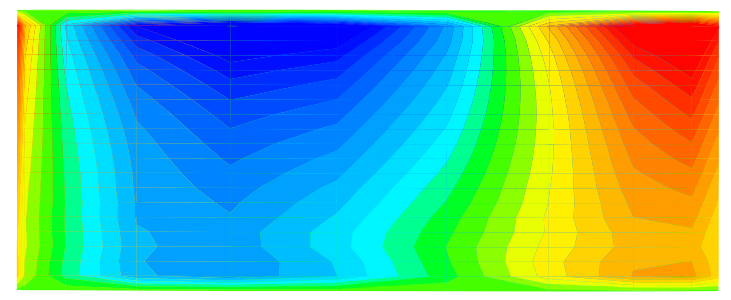

(b): numerical, heating from above is $\mathrm{m}=2$ for a bridge heated from above and $\mathrm{m}=1$ for a bridge heated from below.

From the number of temperature disturbances the difference between the asymmetric $(\mathrm{m}=1)$ and the symmetric $(\mathrm{m}=2)$ flow instabilities is evident.

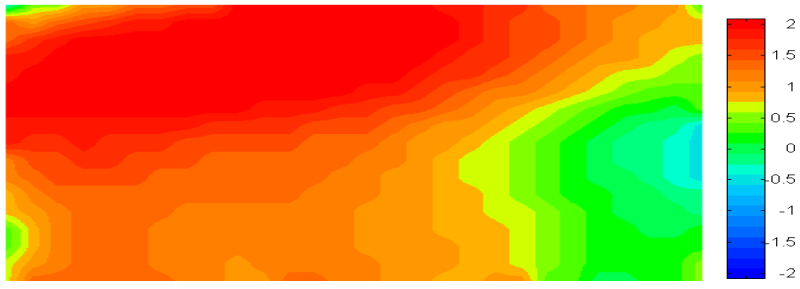

(d): experimental, heating from below

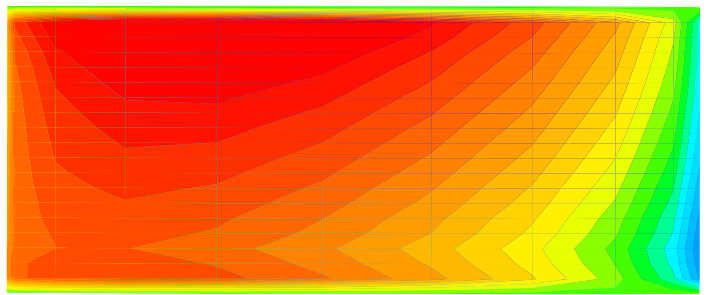

(e): numerical, heating from below 


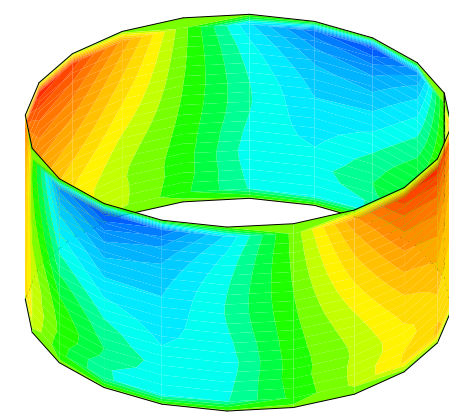

(c): numerical, heating from above(3D view)

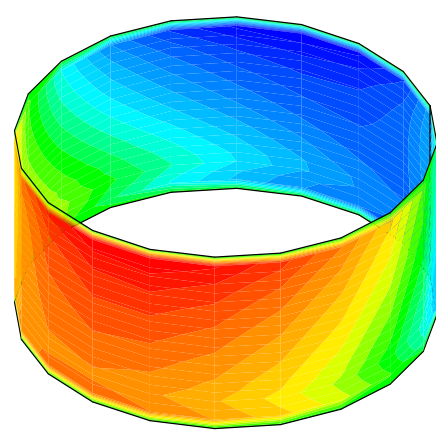

(f): numerical, heating from below (3D view)

Fig. 16-Surface temperature disturbances in the case $\mathrm{A}=0.5$, heating from above $\left(\mathrm{Ma}=3.5 \times 10^{4}\right)$ or from below $\left(\mathrm{Ma}=4.3 \times 10^{4}\right)$, rotating regime.

\section{$\underline{\text { 5. CONCLUSIONS }}$}

Surface tension-driven instabilities in vertical cylindrical liquid bridges have been studied experimentally using a microscale facility and numerically with time-dependent threedimensional simulations. Both the experimental and numerical results have shown the different flow organizations corresponding to the standing wave and to the travelling wave regimes in bridges of different aspect ratios. The azimuth flow organization at the onset of instability depends on the geometrical aspect ratio and may change according to whether the bridge is heated from above or from below. In particular, when the liquid bridge is heated from below, the critical Marangoni number is larger and the critical wave number $m$ can be smaller compared to the case of bridge heated from above.

Acknowledgements - This work was supported by the Italian Space Agency (ASI).

\section{REFERENCES}

[1] C.H. Chun, W. West, "Experiments on the transition from the steady to the oscillatory Marangoni convection of a floating zone under reduced gravity effect", Acta Astronautica 6, 1073 (1979).

[2] R. Monti, "On the onset of the oscillatory regimes in Marangoni flows", Acta Astronautica 15, 557 (1987)
[3] F. Preisser, D. Schwabe, A. Scharmann, "Steady and oscillatory thermocapillary convection in liquid columns with free cylindrical surface", J.Fluid Mech. 126, 545 (1983).

[4] R. Velten, D. Schwabe, A. Scharmann, "The periodic instability of thermocapillary convection in cylindrical liquid bridges", Phys.Fluids A 3, 267 (1991)

[5] R. Monti et al., in "Summary Review of Sounding Rockets Experiments in Fluid Science and Material Science", ESA-SP1132, Vol. 1-4

[6] R. Monti, C. Albanese, L. Carotenuto, D. Castagnolo, E. Ceglia 1994, "First Results from Onset experiment during D2 Spacelab Mission", Symposium on Scientific Results of the German Spacelab Mission D2 Norderney, March, 1994

[7] A. Hirata, S. Nishizawa, M. Sakurai, "Experimental results of oscillatory Marangoni convection in a liquid bridge under normal gravity", J. Jpn. Soc. Microgravity Appl., 14, 122 (1997)

[8] R. Savino, R. Monti, "Oscillatory Marangoni convection in cylindrical liquid bridges", Phys. Fluids, 8, 2906 (1996)

[9] R. Monti, R. Savino, M. Lappa, "Oscillatory Thermocapillary flows in simulated floating zones with time-dependent boundary conditions"; 47th IAF Congress (1996), in press on Acta Astronautica .

[10] R. Monti, R. Savino, M. Lappa and R. Fortezza,"Activities in preparation of the sounding rocket experiment on oscillatory Marangoni flow" 48th IAF Congress (1997). 